\title{
A MATHEMATICAL MODEL OF SOLID-STATE DEWETTING OF BARIUM THIN FILMS ON $W(112)$
}

\author{
S.A. Knavel ${ }^{1}$, T.V. Savina ${ }^{1, *}$, M.V. Mroz ${ }^{2}$, M.E. Kordesch ${ }^{2}$, \\ C.N. EAds ${ }^{3}$, J.T. SAdOWSki ${ }^{3}$ And S.A. TEnNEY ${ }^{3}$
}

\begin{abstract}
Solid state dewetting occurs when a thin solid film is heated. The temperature of dewetting depends on the thickness of the film; dewetting can be observed in the range of $1 / 3$ to $1 / 2$ of the bulk melting temperature. While remaining solid, the film behaves in a manner similar to liquids dewetting and agglomerating to forming islands or droplets. One of the possible mechanisms is the conversion of a metastable thin film geometry into a more stable form. Heating the metastable film gives the film atoms higher mobility, and the films retract, dewetting the surface. This atomic motion can be restricted due to surface anisotropy. We present in situ emission microscopy observations of barium thin films deposited onto $W(112)$ by thermal evaporation. From the modeling viewpoint, the evolution of the film in this system could be divided in four stages: (i) the nucleation and growth of the thin film as a simply connected region; (ii) formation of droplets/islands/hillocks; (iii) nucleation of holes; (iv) evolution of the components of the disconnected film to their equilibrium state. We present a continuum model that is qualitatively consistent with the evolution of the film observed at the initial stage of the experiment and discuss the later stages of the evolution of surface structures.
\end{abstract}

Mathematics Subject Classification. 35G20, 74H10, 74H15.

Received March 1, 2019. Accepted September 13, 2019.

\section{INTRODUCTION}

The process of uncovering of the substrate initially covered by a continuous film is known as dewetting or agglomeration. It is a thermally activated process leading to a reduction of the film-substrate energy. Dewetting can occur below the film melting temperature. Stresses within the film as well as the energy of the film-substrate interface are typical driving forces of dewetting.

Solid-state dewetting has been observed in different systems including the barium film on a tungsten substrate considered in this paper. The primary driving mechanism is surface diffusion-controlled mass transport at temperatures well below (say, <2/3) the melting point of the film $[1,11,12,17,19]$.

Keywords and phrases: Solid-solid thin film dewetting, barium, $W(112)$.

${ }^{1}$ Department of Mathematics, Ohio University, Athens, OH 45701, USA.

2 Department of Physics and Astronomy, Ohio University, Athens, OH 45701, USA.

3 Center for Functional Nanomaterials, Brookhaven National Laboratory, Upton, NY 11973, USA.

* Corresponding author: savin@ohio.edu 
It is known [17] that solid-state dewetting is affected by the film thickness, the surface self-diffusivity, the energy differences of the film-vapor interface, film-substrate interface, substrate-vapor interface, and the anisotropy of the energies.

From the modeling perspective, solid-state dewetting for a number of systems, when the film thickness is greater than $10 \mathrm{~nm}$, goes through two stages [13]. An example is gold on fused silica substrates [7]. During the first stage, the film-vapor interface starts to evolve from the initially flat position into a more complicated shape, however the film remains connected. Hole and void nucleation occurs during this stage. At the second stage, holes grow and the film becomes disconnected by forming isolated islands. The processes occurring during the latter stage are edge retraction, the growth of rims on retracting edges, the break-up of rims to form strands, and the break-up of strands to form islands. The retraction of film edges and the formation of thickened rims along the edges is due to the capillary-driven surface diffusion on the film-vapor interface. In some systems, the rims grow with time, and either impinge on each other and coalesce into larger clusters or allow the fingering instability [17], which results in strands of material. Next, the strands break up due to a Rayleigh-Plateau instability, leading to the formation of islands/particles of material. As it was mentioned in [21], the shapes of holes in the film play an important role in determining the final distribution of islands. Thus, understanding the morphological evolution of holes is essential for controlling the dewetting process as a whole [21].

It is also known from the literature that sometimes dewetting happens as a "fractal growth" [2], when a small hole nucleates at an area with high stress creating "branches of holes" during further annealing. It was conjectured in [19], that fractal growth occurs when both film-vapor and film-substrate interfaces become unstable rather than when only the film-vapor interface is unstable.

Solid-state dewetting can be modeled by a higher-order partial differential equation in a domain with a moving boundary (see [19] for the corresponding conditions on the moving interface in 1D). The authors of [19] considered a single cylindrical hole in the flat film and reported three different dynamics: the hole shrinks, the hole dewets (becomes large), and creation of a void when the top layer is continuous but the film is detached from the substrate.

The aim of this paper is to study the initial stage of the film evolution; we report some recent experimental results and attempt to formulate a mathematical model. This study was motivated by recent advances in thermionic electron emitters that highlight the structure of the emitting surface, and its composition. Barium is the key ingredient for achieving a low work function surface and high electron emission density [8].

Dewetting is a self-regulating phenomenon that ensures a thin layer of barium on the surface. Recent theoretical studies [5] find that $\mathrm{Ba}$ is unstable on the clean tungsten low index surfaces, consistent with dewetting. In the work shown by Zhou et al. [20], similar calculations show that barium adsorption on clean tungsten is unstable relative to the Ba-oxides on $W(100), W(111)$ and $W(112)$. In both the experimental work [8], and in the theoretical models, $W(112)$ is found to be an important surface. While we have observed that barium desorbs from $W(100)$ and $W(110)$ [10]; the $W(112)$ single crystal that we have used for the dewetting experiment is the one with the best prepared surface. On surfaces with defects and step bunches, the dewetting can be influenced by surface features. The $W(112)$ used in this work is the smoothest and least defective of the crystals that we have prepared.

\section{EXPERIMENTAL RESULTS}

The $W(112)$ crystal was purchased from Princeton Scientific Corp., Easton PA. The crystal is $10 \mathrm{~mm}$ in diameter and $2 \mathrm{~mm}$ thick. It was polished to less than 0.01 micron surface roughness and to within 0.1 degree of the (112) surface by the vendor. Standard cleaning methods were used: Ar ion sputtering and repeated heating in $5 \times 10^{-7}$ Torr $\mathrm{O}_{2}$ at approximately $1500^{\circ} \mathrm{C}$ followed by flashing to approximately $1500{ }^{\circ} \mathrm{C}$ in vacuum. The barium was deposited from a tungsten filament wound into a spiral of 5 turns, $4 \mathrm{~mm}$ in diameter, using tungsten wire of $0.37 \mathrm{~mm}$ thickness. The barium coverage was determined by measuring the barium film thickness using a quartz crystal thickness monitor on a separate chamber using the identical deposition geometry and deposition conditions. The deposition conditions used correspond to a $20 \mathrm{~nm}$ thick barium film. The sample temperature 

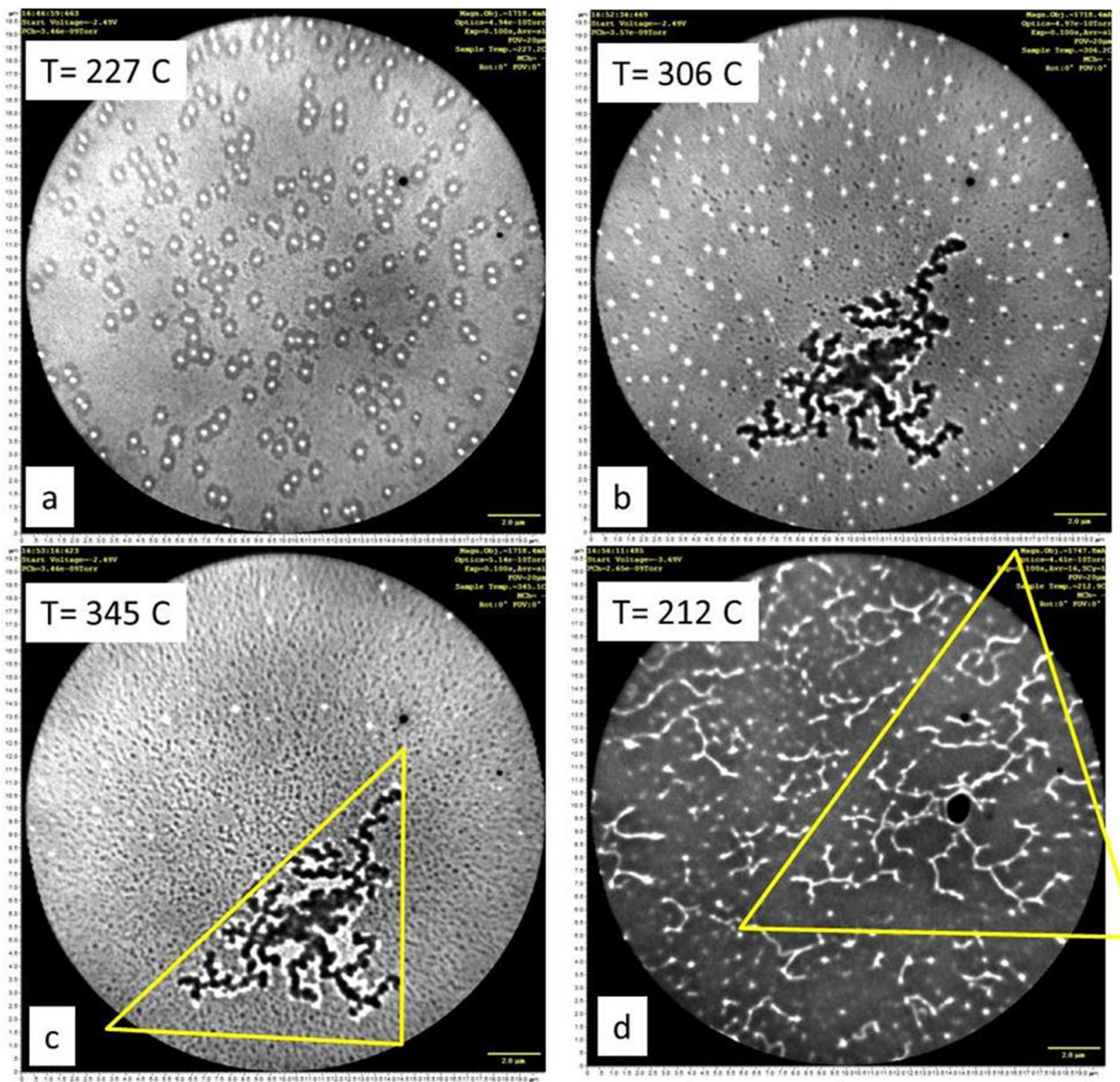

Figure 1. Dewetting of a $20 \mathrm{~nm}$ thick barium film on $W(112)$. The field of view in each image is $20 \mu \mathrm{m}$. Temperatures are given in each image. (a) Droplets with darker rings around them. (b) The dark rings have expanded and are no longer visible in the image. The fractal dewetting area is observed in the lower right of $1 \mathrm{~b}$ ). (c) The droplet number has decreased due to evaporation. (d) The surface at a later time, cooled to $212{ }^{\circ} \mathrm{C}$, and at a different focus. The dewetted barium film is present as elongated ridges and droplets.

was measured using a type $\mathrm{C}$ thermocouple attached to the sample holder in the microscope. The microscope was an Elmitec LEEM V at the Center for Functional Nanomaterials at Brookhaven National Laboratory. This microscope has been used for dewetting studies of barium and scandium on $W(100)$ [8, 10-12]. 

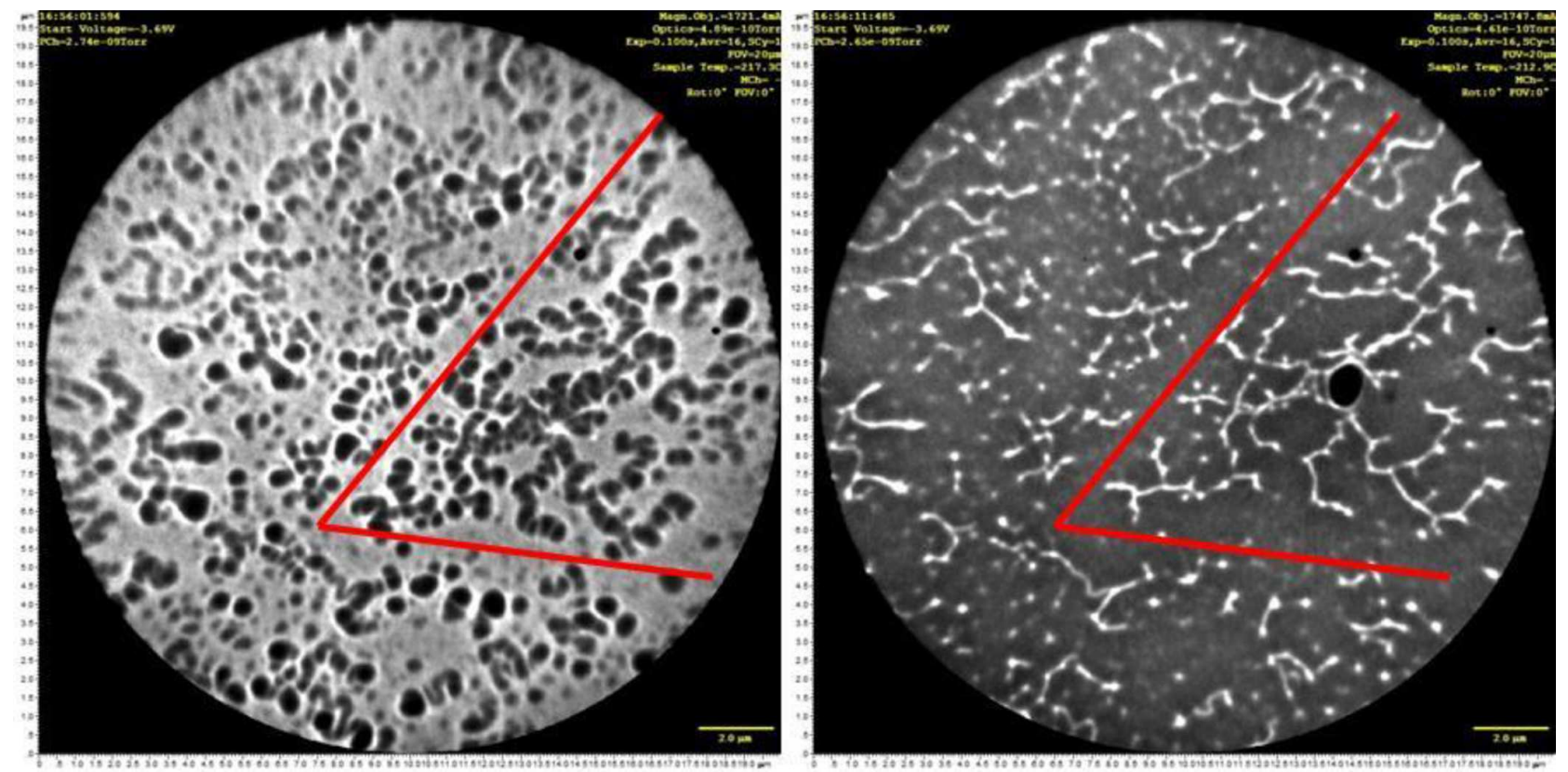

Figure 2. The same region of the surface as in Figure 1d, at two different focus conditions. The red lines show the fractal dewetting area of Figure 1c.

The solid-solid dewetting of barium on $W(112)$ is shown in Figure 1. The imaging mode used is a combination of mirror microscopy and electron reflection microscopy. The work function difference between barium and tungsten is almost $2 \mathrm{eV}$, so that surface fields can play an important role in the focal point of the image when barium islands/droplets are adjacent to clean tungsten areas on the surface. There are most likely at least two barium layers adsorbed on the tungsten surface: one in direct contact with the tungsten, which was observed in [8], and a second layer that is composed of barium on barium. The barium film could be as thick as 40 layers based on the barium lattice constant.

Figure 1 shows reflection-mirror images of the dewetting of a $20 \mathrm{~nm}$ thick barium film deposited on the tungsten (112) surface. In the top left of the image, Figure 1a there are bright spots that are interpreted as droplets/pyramids/islands $\left(T=227^{\circ} \mathrm{C}\right)$. The darker rings around the bright spots are interpreted as thinner areas. The material that was in these thinner areas has been absorbed into the droplet. The dark rings around the bright spots spread over the surface in Figure $1 \mathrm{~b}$ as the surface is heated, until they are no longer observed in the image $\left(T=306^{\circ} \mathrm{C}\right)$ and only the bright spots remain. In Figure 1c, the bright spots decrease in number, indicating that the droplets are evaporating $\left(T=345^{\circ} \mathrm{C}\right)$. The vapor pressure of barium is about $1 \times 10^{-8}$ Torr at $300^{\circ} \mathrm{C}[16]$.

In Figure $1 \mathrm{~b}$ and $\mathrm{c}$, the bottom right hand portion of the image shows a "fractal" feature that grows with increasing time and temperature. Although this part of the image is dark in Figure 1b-d, the film geometry is a ridge of barium metal on the surface. The extension of this dark feature over the entire surface can be seen in Figure 1d. A comparison of this region of the sample at a different focus is shown in Figure 2. The dark spots have been reversed to bright areas, due to a change in the image focus.

Due to the distortions of the surface electric field by the barium layers and droplets, the interpretation of light and dark objects in the image can be difficult. Figure 2 shows that an object that appears bright in the image at a given focus can be changed to a dark black object at different focus. 


\section{MATHEMATICAL MODEL}

\subsection{The governing equation for evolution of the connected thin film}

We consider the following equation as a governing equation describing the evolution of the film-vapor interface $h(x, y, t)$ during the initial stage of the annealing when the film remains connected,

$$
\frac{\partial h}{\partial t}(x, y, t)=\nabla^{2}\left(\nabla^{4} h+\nabla^{2} h-\left(\nabla \cdot P_{3}\right)\right)-\mu|\nabla h|^{2}
$$

where

$$
P_{3}=|\nabla h|^{2} \nabla h+\frac{b-1}{2}(\nabla h \cdot \mathbf{E} \nabla h) \mathbf{E} \nabla h .
$$

Here $\mathbf{E}$ is the permutation matrix, $\mu$ is the evaporation rate, and $b$ is the anisotropy coefficient. Equation (3.1) was derived from the Mullins's phenomenological model [13], stating that the normal velocity of the moving interface, $v_{n}$, is proportional to the surface Laplacian acting on the corresponding chemical potential (see $[3,15]$ ). The first term in the right-hand side of (3.1) describes the reconstruction of the surface due to surface diffusion along the film-vapor interface, morphological instability, and surface tension. The second term models the effect of evaporation of the film with a rate $\mu$.

A one-dimensional version of this equation reduces to

$$
\frac{\partial h}{\partial t}(x, t)=\frac{\partial^{2}}{\partial x^{2}}\left(\frac{\partial^{4} h}{\partial x^{4}}(x, t)+\frac{\partial^{2} h}{\partial x^{2}}(x, t)-3\left(\frac{\partial h}{\partial x}(x, t)\right)^{2} \frac{\partial^{2} h}{\partial x^{2}}(x, t)\right)-\mu\left(\frac{\partial h}{\partial x}(x, t)\right)^{2}
$$

Differentiating the latter equation with respect to $x$ and replacing the slope $h_{x}:=\frac{\partial h}{\partial x}$ with $p$, we have

$$
p_{t}=\left(p_{x x}+p-p^{3}\right)_{x x x x}-\mu p p_{x} .
$$

\subsection{Effect of the evaporation rate on the stationary solutions}

In this section we study the effect of evaporation on the stationary solutions of equation (3.3) by varying the coefficient $\mu$.

We remark that function $p_{0}(x)= \pm \tanh (x / \sqrt{2})$ is a solution to the equation $p_{x x}+p-p^{3}=0$ and, therefore, it satisfies the stationary version of (3.3),

$$
\left(p_{x x}+p-p^{3}\right)_{x x x x}-\mu p p_{x}=0
$$

for the special case when $\mu=0$. The corresponding antiderivative $h_{0}(x)=\int p_{0} d x= \pm \sqrt{2} \ln \cosh (x / \sqrt{2})$ models a pit or a hill depending on the sign.

Next, we aim to show that a nonzero evaporation affects the hills and the pits by making the pits bigger and the hills smaller. This illustrates our interpretation of the bright spots in Figure 1 as droplets/pyramids/hillocks.

Analogously to the analysis performed in [15] for the case of the normal deposition, we integrate the equation (3.4) with respect to $x$, obtaining

$$
\left(p_{x x}+p-p^{3}\right)_{x x x}+\frac{\mu}{2}\left(A^{2}-p^{2}\right)=0 .
$$

Here $A$ is a constant of integration, which corresponds to the value of the slope $p$ far from the vertex. 
Linearizing $p(x)$ for large positive values of $x$ about $A, p(x)=A+\widetilde{p}(x)$, we have

$$
\left(\widetilde{p}_{x x}+\left(1-3 A^{2}\right) \widetilde{p}\right)_{x x x}-\mu A \widetilde{p}=0
$$

A general solution to this equation is a linear combination $\widetilde{p}=\sum_{1}^{5} a_{i} e^{\sigma_{i} x}$ with arbitrary coefficients $a_{i}$, where $\sigma_{i}$ are defined by the polynomial $\sigma^{5}+\left(1-3 A^{2}\right) \sigma^{3}-\mu A=0$. Since we look for solutions taking values close to $A$ at infinity, and, therefore, bounded at infinity, the coefficients, $a_{i}$ of the eigenvalues $\sigma_{i}$ with positive real parts, must vanish. Let us find the number of such eigenvalues.

In the following analysis, we assume that the evaporation rate $\mu$ is a small positive constant. The eigenvalues $\sigma_{i}$ depend on the signs of $A$ and $\widetilde{A}=3 A^{2}-1$.

Thus, in the leading order we have

- When both $A$ and $\widetilde{A}$ are positive,

$$
\sigma_{1,2}= \pm \widetilde{A}^{\frac{1}{2}}+\frac{A}{2 \widetilde{A}^{2}} \mu+o(\mu), \quad \sigma_{3,4,5}=\left(\frac{\mu A}{\widetilde{A}}\right)^{1 / 3} e^{\frac{i(\pi+2 \pi k)}{3}}+o\left(\mu^{\frac{1}{3}}\right), \quad k=0,1,2,
$$

which indicates that there are three eigenvalues with positive real parts. Note that this case corresponds to a positive kink, $p_{0}=\tanh (x / \sqrt{2})$.

- When $A<0$ and $\widetilde{A}>0$,

$$
\sigma_{1,2}= \pm \widetilde{A}^{\frac{1}{2}}-\frac{|A|}{2 \widetilde{A}^{2}} \mu+o(\mu), \quad \sigma_{3,4,5}=\left(\frac{\mu|A|}{\widetilde{A}}\right)^{1 / 3} e^{\frac{2 \pi i k}{3}}+o\left(\mu^{\frac{1}{3}}\right), \quad k=0,1,2
$$

Two of these eigenvalues have positive real parts. This corresponds to the negative kink, $p_{0}=$ $-\tanh (x / \sqrt{2})$.

- When both $A$ and $\widetilde{A}$ are negative,

$$
\sigma_{1,2}= \pm i|\widetilde{A}|^{\frac{1}{2}}-\frac{|A|}{2 \widetilde{A}^{2}} \mu+o(\mu), \quad \sigma_{3,4,5}=\left(\frac{\mu|A|}{|\widetilde{A}|}\right)^{1 / 3} e^{\frac{i(\pi+2 \pi k)}{3}}+o\left(\mu^{\frac{1}{3}}\right), \quad k=0,1,2
$$

which results in two eigenvalues with positive real parts.

- When $A>0$ and $\widetilde{A}<0$,

$$
\sigma_{1,2}= \pm i|\widetilde{A}|^{\frac{1}{2}}+\frac{A}{2 \widetilde{A}^{2}} \mu+o(\mu), \quad \sigma_{3,4,5}=\left(\frac{\mu A}{|\widetilde{A}|}\right)^{1 / 3} e^{\frac{2 \pi i k}{3}}+o\left(\mu^{\frac{1}{3}}\right), \quad k=0,1,2,
$$

leading to three eigenvalues with positive real parts.

The above analysis indicates that the solution as a single pit is unique, while a solution as a single hill is not unique, and there exists a one-parameter family of hills.

Indeed, a unique solution of equation (3.5), requires six additional conditions. In both cases, a pit and a hill, the slope satisfies $p(0)=p_{x x}(0)=p_{x x x x}(0)=0$. The rest of the conditions could be chosen from continuity, $p(x) \rightarrow p_{0}(x)$ as $\mu \rightarrow 0$. The number of these conditions equals to three, therefore, involves three arbitrary parameters. For instance, the remaining conditions could be listed as $p_{x}(0)=\left(p_{0}\right)_{x}+\eta_{1}(\mu), A= \pm 1+\eta_{2}(\mu)$, and $p_{x x x}(0)=\left(p_{0}\right)_{x x x}+\eta_{3}(\mu)$, where $\lim _{\mu \rightarrow 0} \eta_{i}(\mu)=0, i=1,2,3$. Thus, the solution is uniquely determined by three corrections $\eta_{i}(\mu)$, which could be found from setting $a_{i}\left[\Re \sigma_{i}>0\right]=0$. 

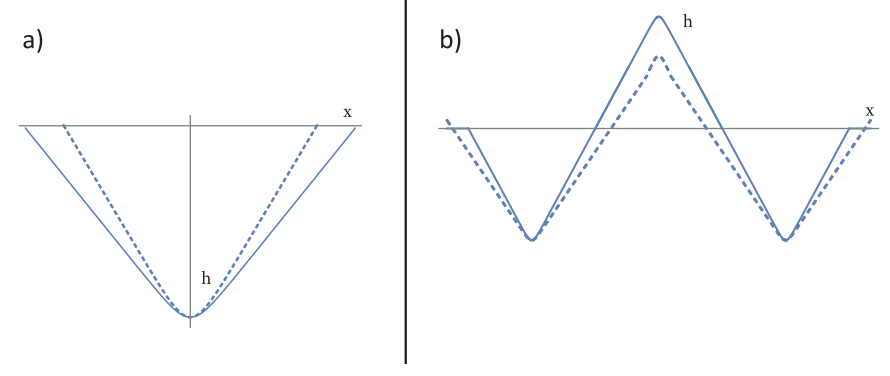

FiguRE 3. An effect of evaporation on the stationary solutions. (a) A single pit: solid line corresponds to $\mu=0$, dashed line corresponds to $\mu=0.1$. (b) A hill surrounded by two pits: solid line corresponds to $\mu=0$, dashed line corresponds to $\mu=0.05$.

\subsection{Construction solutions as a single pit and a hill surrounded by pits}

As it was shown in the previous subsection, the stationary solution of the one-dimensional version of the governing equation as a single pit is unique. Thus, the solution as a hill surrounded by two pits is also uniquely defined. We are interested in such a solution because it resembles a bright spot, surrounded by a darker area in Figure 1. In this section, we construct both of these solutions using the method of matched asymptotic expansions [15].

The uniqueness of the solution as a single pit results in the uniqueness of its slope. To find a correction to the slope due to evaporation with a (small) rate $\mu$, we look for the value of the scalar $\phi_{1}$ in the following expression for the slope of the pit far from the vertex

$$
A=1-\mu^{1 / 3} \phi_{1}+o\left(\mu^{1 / 3}\right)
$$

Note, that function $h(x)$ modeling a pit and function $p(x)$ modeling its slope, are an even and an odd functions respectively, therefore, it is sufficient to evaluate them only for positive values of $x$. We represent the inner solution of (3.5) in the form

$$
p_{\text {in }}(x, \mu)=p_{0}(x)+\mu^{1 / 3} p_{1}(x)+\mu^{2 / 3} p_{2}(x)+\mu p_{3}(x)+\cdots
$$

with unknown functions $p_{i}, i=1,2,3$ and $p_{0}(x)=\tanh (x / \sqrt{2})$. Equation (3.5) can be rewritten in terms of a long-scale variable, $\xi=\mu^{1 / 3} x$, resulting in

$$
\left(P_{\xi \xi}+P-P^{3}\right)_{\xi \xi \xi}-\frac{1}{2}\left(P^{2}-A^{2}\right)=0
$$

Note that the solution to this equation, the outer solution, is an odd function, so it is sufficient to consider only the case when $\xi>0$. The outer solution we write as

$$
P(\xi, \mu)=1+\mu^{1 / 3} P_{1}(\xi)+\mu^{2 / 3} P_{2}(\xi)+\mu P_{3}(\xi)+\cdots,
$$

where functions $P_{i}, i=1,2,3$, are to be found from matching the inner and the outer solution using the method described in [15]. The matching also results in finding the correction $\phi_{1}$. Thus, the slope of the pit for positive values of $x$ could be approximated as $A \approx 1-0.561 \mu^{1 / 3}$. Figure 3a illustrates the effect of evaporation: the decrease of the slope of the pit makes the pit wider. Figure $3 \mathrm{~b}$ shows how $\mu$ affects the evolution of a pit surrounded by hills: while the pits grow with increase of $\mu$, the hills become smaller. 


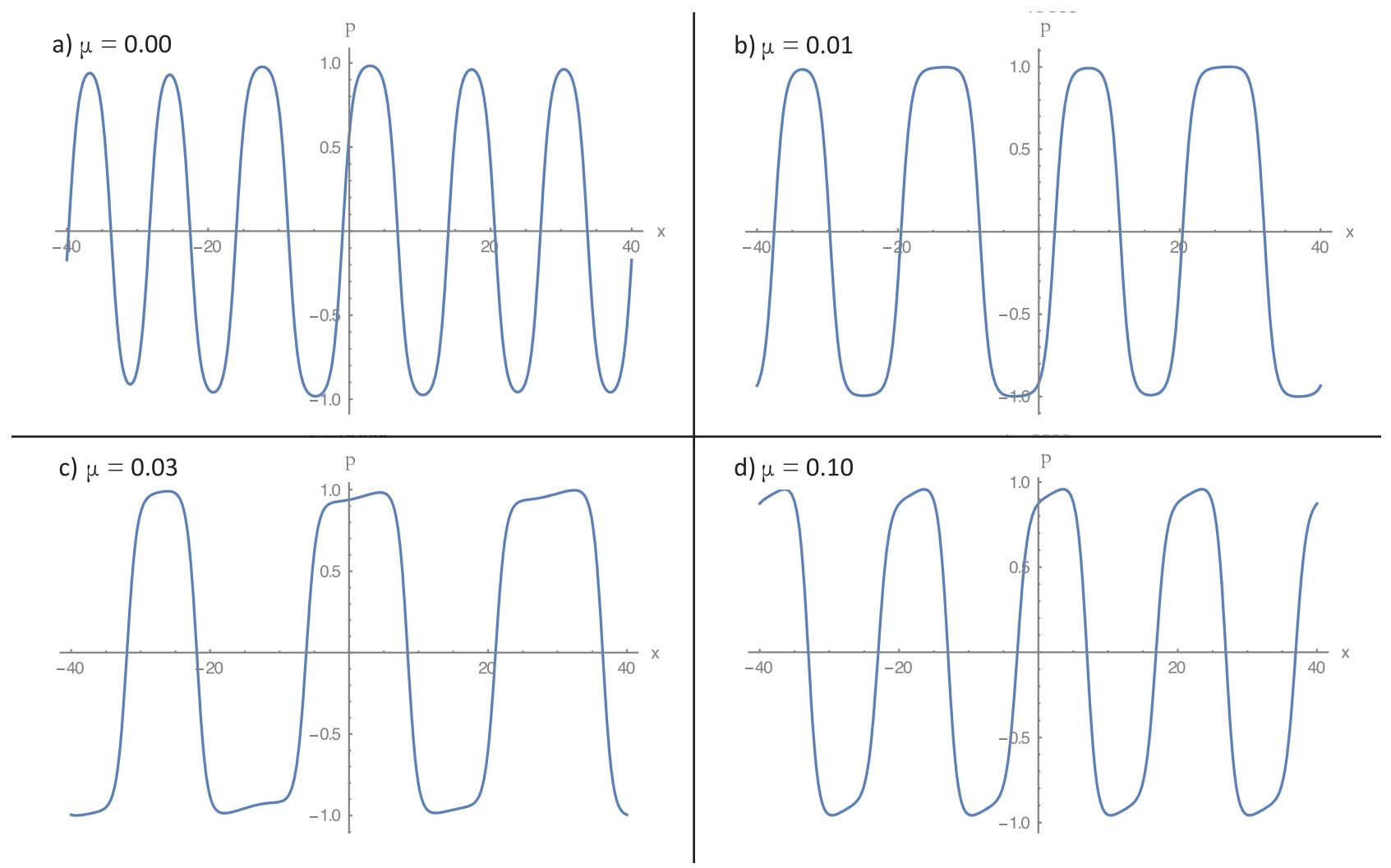

Figure 4. Slope of the film-vapor interface for different values of the evaporation rate.
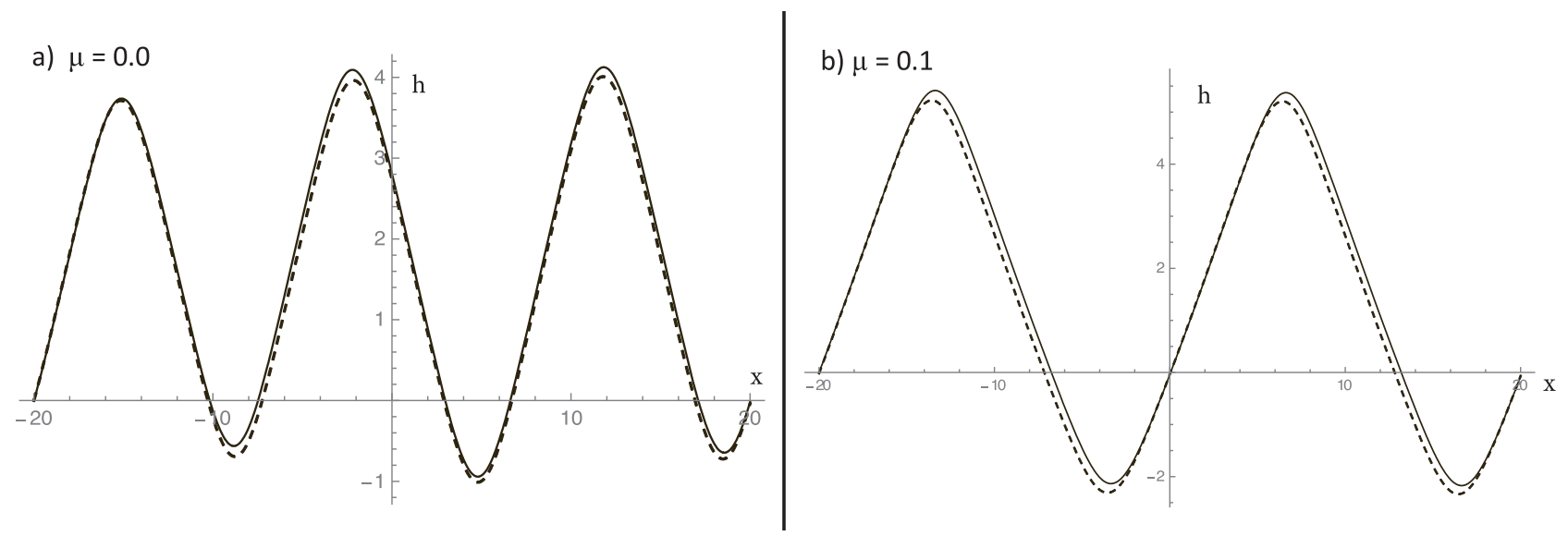

FiguRE 5. Snap-shots of the film-vapor interface for different values of the evaporation rate at an earlier time (solid line) and at a later time (dashed line).

We performed numerical simulations of the boundary value problem for equation (3.3) with periodic boundary conditions. The long-time behavior of the slope, $p(x, t)$, of the film-vapor interface is presented in Figure 4 for different evaporation rates at $t=10^{4}$. The time was chosen with a goal to obtain the stationary (or slowly changing) solutions. The dynamics of the evolution for small values of $\mu$ goes through the following stages 
[15]: first, the solution "forgets" the initial condition by forming a hill-and-valley structure, then the coarsening occurs, finally coarsening stops, which often results in the stationary solutions (see Fig. 4b,d).

The shapes of the interface, $h(x, t)$, are shown in Figure 5 with and without evaporation for two different moments of time $t=0.5 \times 10^{4}$ and $t=10^{4}$.

Figures $4 \mathrm{~b}-\mathrm{d}$ and $5 \mathrm{~b}$ show that the nonzero evaporation rate breaks the symmetry between the slopes of the hillock and pits compared to the case corresponding to $\mu=0$, presented in Figures 4a and 5a. This results in hillocks getting more narrow and pits getting wider (see Fig. 5b).

\section{Discussion}

\subsection{Temperature-dependent evaporation rate}

In the previous section, we considered a model with a constant evaporation rate $\mu$. Taking into account the data known in the literature regarding the temperature-dependence of the evaporation rate of barium $[4,18]$, we include this dependence into our governing equation. Since equation (3.1) depends on time rather than on the temperature, we use the dependence of temperature versus time from our experiment, which suggests that a linear dependence, $\mu=\mu_{0}+\mu_{1} t$, seems to be a reasonable approximation.

We performed numerical simulations of equation (3.2) with periodic boundary conditions. Figure 6 shows the film evolution with $\mu_{0}=0.01$ and $\mu_{1}=0.001$. The time in Figure $6 \mathrm{a}$ corresponds to the moment when the droplets/hillocks were just formed. These hillocks disappear at a later time (see Fig. 6d). The dynamic with the temperature-dependent rate $\mu$ is essentially different compared with a constant rate. In this case, the coarsening does not play a role in the process. A conclusion regarding whether or not the coarsening is important for the evolution of the film could be made after more careful observation of the initial stage of the film dewetting in the future experiments.

Remark that the equation (3.1) models the earlier stage of the evolution of a barium film when the depth of the pits is smaller than the film thickness. To model the later stages of dewetting, our model needs an adjustment. One of the ideas known from the literature is discussed in the next subsection.

\subsection{Growth of holes}

In this paper we were mostly concerned with the initial stage of evolution of Ba thin film leading to the nucleation of the holes via growth of pits.

Once a pit reaches the substrate it forms a hole, which starts to grow after reaching a critical size. The rate of this growth is defined by the rate at which the edge of the film retracts. This retraction is governed by capillary energies [17]. When an edge retracts, in many systems it forms a rim followed by a valley. However, in some systems the hole propagation occurs only on the parts of the layer that have the same thickness as the pristine film [6]. The curvature of the film close to the edge is high. The driving forces of the mass transfer may be due to evaporation-condensation mechanisms as well as surface diffusion [17]. Both processes are included into the governing equation (3.1). The hole growth of a single cylindrical hole was considered in [14], where the authors mention that a hole may either grow or shrink. In addition to those two scenarios, the authors of [19] mention the third possible scenario, when the top layers of the film are connected leaving a void underneath.

Dewetting exhibits different morphologies depending on the system [6]. The holes can show fractal features (see the bottom part of Fig. 1b,c). That was observed in other systems [9], where it was interpreted as a Diffusion Limited Aggregation (DLA), a rigorous theory of which is still an open problem.

The growth model for a single cylindrical hole was suggested in [14], where the authors estimated the asymptotic velocity of the hole growth. Taking into account the cylindrical symmetry, the continuum model in [14] was reduced the the following one-dimensional equation for $h(r, t)$, where $r=\left(x^{2}+y^{2}\right)^{1 / 2}$ :

$$
\frac{\partial h}{\partial t}(r, t)=\Delta_{r}\left(\Delta_{r} h(r, t)\right),
$$




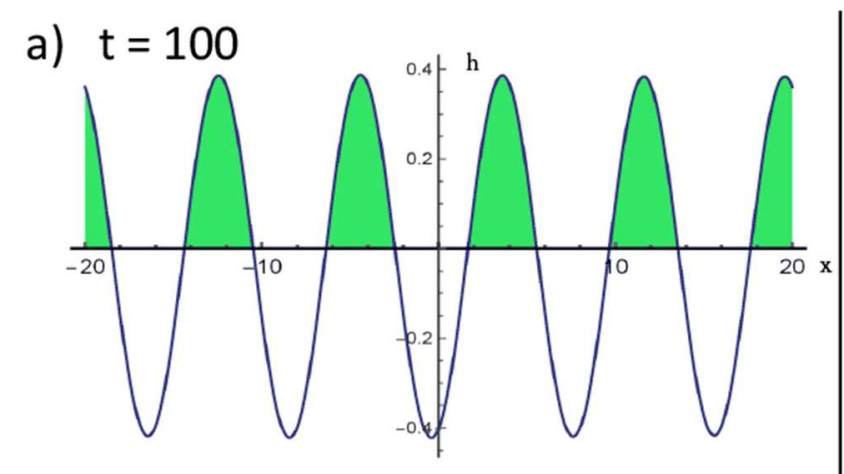

b) $t=800$
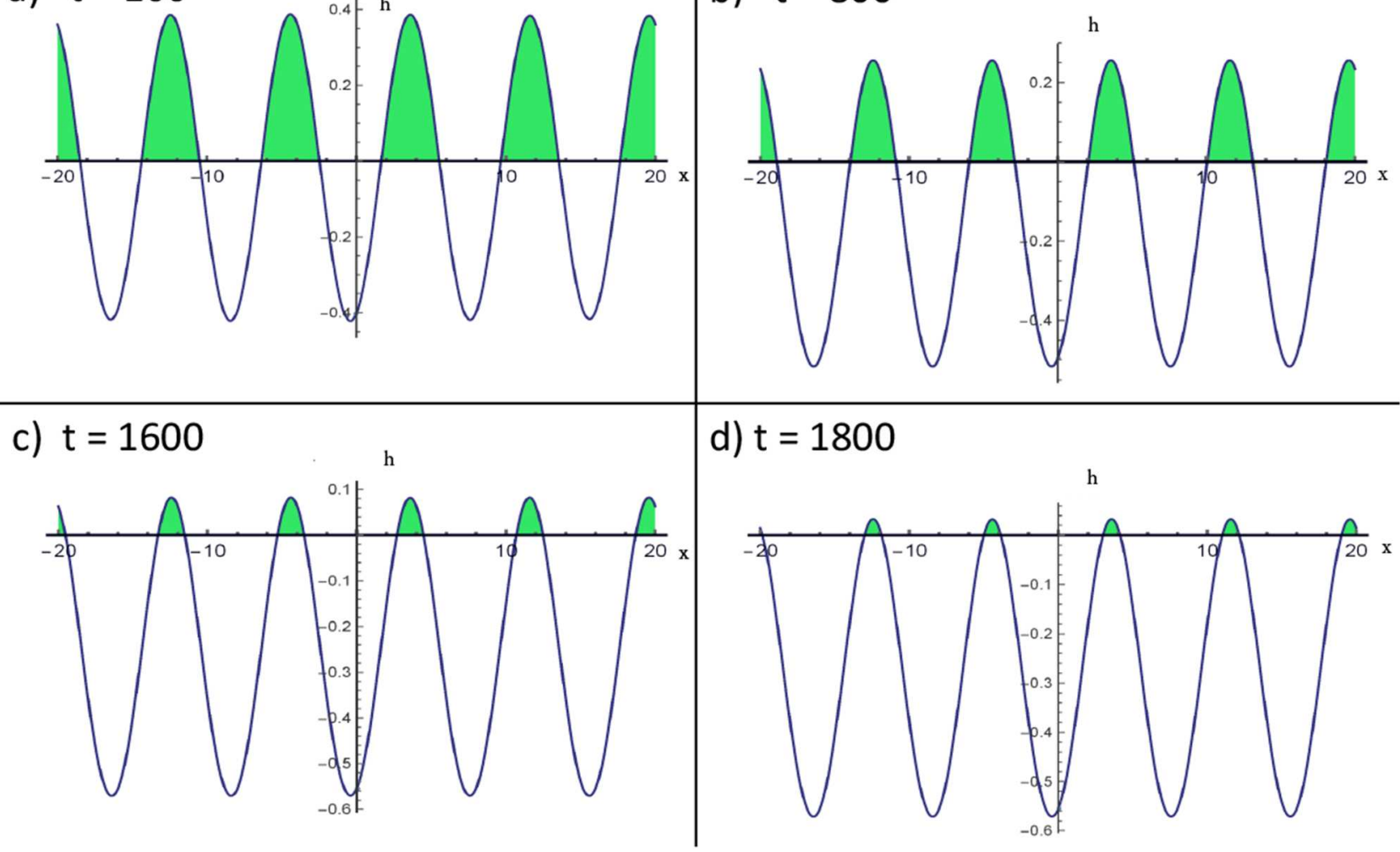

d) $t=1800$

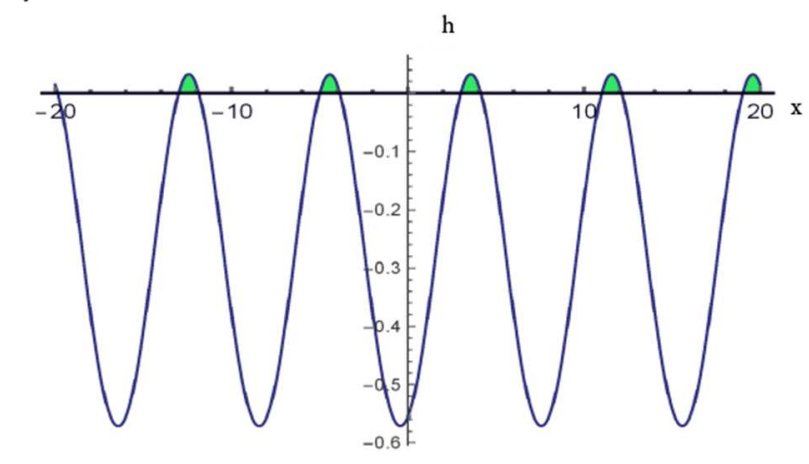

FiguRE 6. Snap-shots of the film-vapor interface at different times, $t$, for a temperaturedependent evaporation rate. The $x$-axis corresponds to the initial position of a flat film.

where

$$
\Delta_{r}=\frac{\partial^{2}}{\partial r^{2}}+\frac{1}{r} \frac{\partial}{\partial r}
$$

The equation (4.1) is complemented with the following boundary conditions with the assumption that $h=0$ corresponds to the film-vapor interface and $h=-h_{0}$ corresponds to the film-substrate interface,

$$
\begin{gathered}
h(R, t)=-h_{0}, \\
h_{r}(R, t)=\tan \theta, \\
h_{r r r}(R, t)+\frac{1}{R} h_{r r}(R, t)-\frac{1}{R^{2}} h_{r}(R, t)=0, \\
h(\infty, t)=0,
\end{gathered}
$$

where $h_{0}$ is the film thickness, $R$ is the radius of the cylindrical hole centered at the origin, and $\theta=\arccos \left(\left(\gamma_{s v}-\right.\right.$ $\left.\gamma_{s f}\right) / \gamma_{v f}$ ) is the equilibrium contact angle. Here $\gamma_{v f}$ is the surface tension of the film-vapor (or vacuum) interface, 
$\gamma_{s v}$ is the surface tension of substrate-vapor interface, and $\gamma_{s f}$ is the substrate-film surface tension. This model [14] suggests that velocity of hole growth vanishes with time as $t^{-3 / 4} \ln ^{3}\left(t / R_{c}^{4}\right)$, where $R_{c}=h_{0} / \tan \theta$.

\section{Conclusions}

We studied a dynamic of barium thin film deposited onto a tungsten substrate. From our observation we infer that the evolution is going through the following stages: at the initial stage we observed formation of hillocks (lighter spots in Fig. 1) surrounded by pits (darker areas in Fig. 1). The darker areas grow with time. Then the hillocks disappear and the pits reach the substrate. After that the holes grow demonstrating some fractal growth, which could be interpreted as diffusion-limited aggregation. We suggested a model that qualitatively agrees with our observations of the evolution of the barium film at the initial stage. Our model explains the formations of hillocks and pits as well as the growth of the pits and the elimination of the hillocks.

Acknowledgements. This work was supported by DARPA INVEST grant number N66001-16-1-4040. This research used resources of the Center for Functional Nanomaterials, which is a U.S. DOE Office of Science Facility, at Brookhaven National Laboratory under Contract No. DE-SC0012704.

\section{REFERENCES}

[1] R. Brandon and F.J. Bradshaw, The mobility of the surface atoms of copper and silver evaporated deposits in Technical Report 66095. Royal Aircraft Establishment, Farnborough (1966).

[2] P.R. Gadkari, A.P. Warren, R.M. Todi, R.P. Petrova and K.R. Coffey, Comparison of the agglomeration behavior of thin metallic films on $\mathrm{SiO}_{2}$. J. Vac. Sci. Technol. A 23 (2005) 1152-1161.

[3] A.A. Golovin, M.S. Levine, T.V. Savina and S.H. Davis, Faceting instability in the presence of wetting interactions: a mechanism for the formation of quantum dots. Phys. Rev. B $\mathbf{7 0}$ (2004) 235342.

[4] K.T. Jacob and Y. Waseda, The vapour pressure of barium and strontium. J. Less-Common Metals 139 (1988) $249-259$.

[5] R. Jacobs, D. Morgan and J. Booske, Work function and surface stability of tungsten-based thermionic electron emission cathodes. APL Mater. 5 (2017) 116105.

[6] P. Jacquet, R. Podor, J. Lautru, J. Teissere, I. Gozhyk, J. Jupille and R. Lazzari, On the solid-state dewetting of polycrystalline thin films: Capillary versus grain growth approach. Acta Mater. 143 (2018) 281-290.

[7] E. Jiran and C.V. Thompson, Capillary instabilities in thin films. J. Electr. Mater. 19 (1990) 1153-1160.

[8] D.M. Kirkwood, S.J. Gross, T.J. Balk, M.J. Beck, J. Booske, D. Busbaher, R. Jacobs, M.E. Kordesch, B. Mitsdarffer, D. Morgan, W.D. Palmer, B. Vancil and J.E. Yater, Frontiers in Thermionic Cathode Research. IEEE Trans. Electr. Devices 65 (2018) 2061.

[9] J.Y. Kvon, T.S. Yoon, K.B. Kim and S.H. Min, Comparison of the agglomeration behavior of Au and Cu films sputter deposited on silicon dioxide. Appl. Phys. 93 (2001) 3270.

[10] M. Mroz, M.E. Kordesch, J. Sadowski and W. Sitaputra, De-wetting of Barium on W(100) and (110) Surfaces observed using Thermionic Emission Microscopy. 30th International Vacuum Nanoelectronics Conference (IVNC), edited by C. Langer, R. Lawrowski, Book Series: International Vacuum Nanoelectronics Conference (2017) 264-265.

[11] M. Mroz, T. Savina, M.E. Kordesch and S. Tenney, Thermionic emission microscopy of scandium thin film dewetting on $\mathrm{W}(100)$. AIP $A d v .8$ (2018) 065114

[12] M. Mroz, T. Savina, M.E. Kordesch, J.T. Sadowski and S.A. Tenney, Solid Solid dewetting of scandium thin films on the W(100) surface using emission microscopy. J. Vac. Sci. Technol. B 37 (2019) 012903.

[13] W.W. Mullins, Theory of thermal grooving. Appl. Phys. 28 (1957) 333-339.

[14] S.A. Safran and D.J. Srolovitz, Kinetics of instabilities in solid films. Europhys. Lett. 2 (1986) 61-66.

[15] T.V. Savina, A.A. Golovin, S.H. Davis, A.A. Nepomnyashchy and P.W. Voorhees, On faceting of a growing crystal surface by surface diffusion. Phys. Rev. E 67 (2003) 021606.

[16] M. Schmid, Vapor Pressure Calculator. Technik für Menschen, Institut für Angewandte Physik, Wien Surface Physics Group (2013-2018). Available at: https://www.iap.tuwien.ac.at/www/surface/vapor_pressure (2020).

[17] C.V. Thompson, Solid-state dewetting of thin films. Annu. Rev. Mater. Res. 42 (2012) 399-434.

[18] J. Valo and M. Leskela, Thermal analysis in Studies of high- $T_{c}$ superconductors, in Vol. 2 of Handbook of Thermal Analysis and Calorimetry (2003) 817-879.

[19] Y. Wang, W. Jiang, W. Bao and D.J. Srolovitz, Sharp interface model for solid-state dewetting problems with weakly anisotropic surface energies. Preprint arXiv:1407.8331v1 (2014).

[20] Q. Zhou, X. Liu, T. Maxwell, B. Vancil, T.J. Balk and M.J. Beck, $B a_{x} S c_{y} O_{z}$ on W(001), (110), and (112) in scandate cathodes: connecting to experiment via $\mu_{O}$ and equilibrium crystal shape. Appl. Surf. Sci. 458 (2018) 827-838.

[21] R.V. Zucker, G.H. Kim, J. Ye, W.C. Carter and C.V. Thompson, The mechanism of corner instabilities in single-crystal thin films during dewetting. J. Appl. Phys. 119 (2016) 125306. 